\title{
Creation and Evaluation of Emotion Expression with Body Movement, Sound and Eye Color for Humanoid Robots
}

\author{
Markus Häring, Nikolaus Bee and Elisabeth André
}

\begin{abstract}
The ability to display emotions is a key feature in human communication and also for robots that are expected to interact with humans in social environments. For expressions based on Body Movement and other signals than facial expressions, like Sound, no common grounds have been established so far. Based on psychological research on human expression of emotions and perception of emotional stimuli we created eight different expressional designs for the emotions Anger, Sadness, Fear and Joy, consisting of Body Movements, Sounds and Eye Colors. In a large pre-test we evaluated the recognition ratios for the different expressional designs. In our main experiment we separated the expressional designs into their single cues (Body Movement, Sound, Eye Color) and evaluated their expressivity. The detailed view at the perception of our expressional cues, allowed us to evaluate the appropriateness of the stimuli, check our implementations for flaws and build a basis for systematical revision. Our analysis revealed that almost all Body Movements were appropriate for their target emotion and that some of our Sounds need a revision. Eye Colors could be identified as an unreliable component for emotional expression.
\end{abstract}

\section{INTRODUCTION}

Emotion expression is an important feature in human communication. As we aim for the application of robotic systems in social contexts, like health care or education, robots should be able to express emotions to some extend.

A lot of research concerning emotion expression with virtual characters or robots concentrates on facial expression (e.g. [5], [9] or [8]). But designing an expressive face is a huge technical challenge and there is proof that body language is sufficient for this task [2], [7].

In recent years different teams tried to create emotional expressions for robots on different platforms, using quite different approaches. Still there are no validated libraries and no common guidelines on how to design emotional expressions for humanoid robots. Therefore everybody interested in this matter has to design his own expressions anew, at best considering the failures and achievements of his precursors.

Zecca and colleagues [17] combined the bi-pedal robot WABIAN with the expressive face of WE-4RII, an expressive robot with only a torso and head, and so created the whole body expressive robot KOBIAN. Three pools of emotional expression (Anger, Disgust, Fear, Happiness, Surprise, Sadness, Perplexity) were designed: one by students, one by a photographer and one by a cartoonist. They evaluated the recognition ratios and compared the three pools with each other. Their work shows that the problem of creating emotional expressions for a robotic body is not trivial even for experts familiar with the field of emotional expression.
In some cases the student pool even achieved better results than the experts.

Beck and colleagues [2] used the small (about $60 \mathrm{~cm}$ tall) humanoid robot Nao, from Aldebaran Robotics, for their emotional expressions. As Nao has no means for facial expression except the color of its eye LEDs, they limited themselves to emotional key poses (Anger, Sadness, Fear, Pride, Happiness, Excitement). These poses were constructed using recordings from a professional actor and director. They evaluated the effect of a changing head position (up, straight, down) on the identification of emotions and its effect on the three axis of the affect space Arousal-Valence-Stance. They discovered that head position has a strong effect on identification of the displayed emotion and also on the perception of Arousal, Valence and Stance. Their results confirm that a humanoid robot is able to express emotions only with the use of body language, i.e. without facial expressions. And that even small cues (like a different head position) can change the perception of a pose significantly.

Monceaux and colleagues [13] created a library of emotional expressions (40 behaviors related to 15 emotional states) for Nao. For this they did not only use body language but also integrated sounds and differently colored eye LEDs. Their work focuses on the demonstration of the capabilities of Nao for this field of use and the creation of a large pool of different expression. It neither provides a detailed methodology on how to create such expressions nor a form of validation. But their approach extends the work of Beck and colleagues adding movement, sounds and colors.

We created our expressions using the same capabilities of Nao (Body movement, Sound, Eye Color) and validated our work in a rather large pre-test (see II-C). Differently to Zecca and colleagues or Beck and colleagues we did not use the help of professional artists. One difficulty in this approach is the assessment of professional artistic partners. The other is to map their work on robots. Especially the work of Zecca and colleagues shows that this transfer is not always successful.

That is why we tried a different approach and based our expressional designs on psychological research on the connection of emotions to body movement, sound and color. Our expression design is mainly inspired by the work of de Meijer [12], Boone and Cunningham [3] and Coulson [6].

de Meijer's work provides specific data how gross body movements contribute to the attribution of twelve different emotions, including Anger, Fear, Joy and Grief. While some movements are attributed to one specific emotion, others are attributed to a group of related emotions. These findings are 
important guidelines for the design of emotion expression based on body movements.

Boone and Cunningham used this data to create movements using a teddy bear as medium, with which children should express the emotions they associated with pieces of music. Of course we were mostly interested in their movement design, as they study the same emotions as we currently do: Anger, Fear, Sadness and Joy.

Coulson identified in his study six specific postures for Ekman's six basic emotions. Though his postures are static, the different viewpoints of these postures provided valuable information and inspiration for our own expression designs.

Besides the empirically validated emotional expression for the Nao robot using Body Movement, Sound and Eye Color, the novel contribution of our paper is the approach to evaluate the expressivity of the single expressional cues within the Pleasure-Arousal-Dominance model. In this way a detailed analysis is possible, that allows us to check the perception of the components of our expressions with our expectations and the psychological research they are based on. We can identify flaws in our implementation and systematically revise our expressional designs. This approach forms the basis for our effort to create a large pool of validated emotional expressions for the Nao robotic platform as well as the identification and creation of rules and guidelines to combine expressive cues into proper emotional expressions.

\section{EMOTION EXPRESSION WITH BODY MOVEMENT, SOUND AND EYE COLORS}

We created expressions for four different emotions (Anger, Sadness, Fear and Joy), two for each emotion, resulting in eight different expressions (see Figure 1). The Nao V3+ Academic Edition was used as robotic platform and we made use of the three available modalities: Body Movement, Sound and Eye Color (LEDs).

In case of Body Movement we wanted to approximate human behavior, considering Coulsen's static postures and de Meijer's gross body movements.

The connection of sound and emotions is very complex. There is evidence that specific features are important for emotional communication [14]. But Bachorowski and Owren [1], for example, support the perspective that emotional vocal expressions aim more to affect the listener than to express an inner emotional state. We decided to use in most cases human or animal-like acoustic expressions that are commonly related to our emotions, like crying for Sadness, frantic noises for Anger or cheering for Joy. In case of Fear we used other means of acoustical signals, to extend the spectrum of used sounds, thus creating a wider basis for our further research.

\section{A. Expression Design}

First we created the Body Movements. Then we modified our Sounds so they met the timing of the movements. Finally the Eye Colors were added. The behavior modeling software of Nao allowed a simple synchronization of the used modalities, so we did not have to implement our own solution.

\section{1) Anger:}

Anger 1 - in the first expression the robot is furiously gesticulating with its arms while sometimes leaning forward. It is ranting in a gibberish voice, mixed with "Bleeb" sounds known from television when censoring cuss words. The performance ends with the robot raising its arms, shaking, accompanied by rolling thunder (Body Movement $A l$ and Sound A1).

Anger 2 - this expression of anger starts with the robot turning his head to the left side like it would fixate someone. While the body follows the head it clenches its right fist and starts shaking it, like restraining itself. During this performance the eyes are glowing red and the robot is growling like an angry dog (Body Movement A2 and Sound A2).

\section{2) Fear:}

Fear 1 - the robot hectically raises its arms before its head, like protecting itself from something. To emphasize this image he also shies away with its upper body. During this very short and fast movement you hear a loud metallic bang followed by an echo (Body Movement F1 and Sound F1).

Fear 2 - in this expression the robot is cowering, while its whole body is trembling. Inspired by movies in dangerous moments, the robot plays a modulated sound that gets louder (Body Movement F2 and Sound F2).

\section{3) Sadness:}

Sadness 1 - the expression here is quite similar to the one of Fear 2. In this case the robot is not trembling but holds its hands before its face, moving its head from side to side, like wiping its eyes. Appropriately the robot cries, like a small child, fitting the voice pattern of Nao (Body Movement S1 and Sound S1).

Sadness 2 - here the robot is standing in a depressed manner, going limb and crying in its armpit. In this case the voice was extracted from a crying woman and alienated to sound more like a robot. The performance finishes with a sigh and the robot lifts its arms and knees, looking like the robot would take a deep breath (Body Movement $S 2$ and Sound S2).

4) Joy:

Joy 1 - for this expression we made use of the textto-speech module of Nao. The body movement can be best described as a dance of joy, remotely resembling a cheerleader, while the robot is saying "Jippie Yay!" (Body Movement $J$ and Sound J1).

Joy 2 - the robot raises slowly its arms above its head, then pulls them down very fast to its hip, bending its knees a bit. You can describe this gesture as a "winning pose". Appropriately the robot yells "Yehaa", but this time not computed by the text-to-speech module. It is a recording of a man's voice, sounding similar to a cowboy (Body Movement J2 and Sound J2).

\section{B. Eye colors}

Humans cannot change the color of their eyes and thus it is not part of our communication. But there is evidence that colors affect us in an emotional way [16] and hence might be useful to provoke or at least support the perception of 


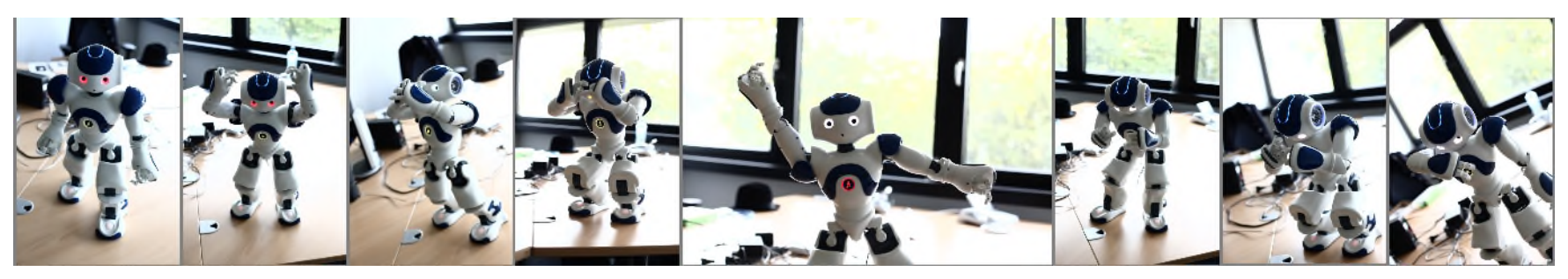

Fig. 1. Emotional Expressions: Anger 1, Anger 2, Fear 1, Fear 2, Joy 1, Joy 2, Sadness 1, Sadness 2

specific emotions. Still choosing colors for emotion expression is a problematic question, as most theories mapping colors to emotions have an esoteric or artistic background and are ambiguous. So we decided to base our choice on examples, that we think are commonly known: In sciencefiction movies aggressive robots are often combined with red glowing eyes. So we thought a viewer would be familiar with this symbol and used it for Anger (Eye color A). In cartoon movies from Disney, sad scenes often have a dark violet tone. In the same way we use a dark violet color for Sadness (Eye color S). Bright and warm colors are often associated with joy and happiness, for example a bright yellow as the color of the sun, which is accordingly our eye color for Joy (Eye color $J$ ). As dark colors are associated with negative emotions (also see [16]) we tried a dark green color (Eye color F) for Fear.

\section{Pre-Test}

Our expressions were presented to a wide audience (children, students, adults and seniors) during an open lap day. The members of the audience were asked to fill in a questionnaire while watching the presentation of the eight emotional expressions. They had to label each expression with one of six options: Anger, Sadness, Fear, Joy, Neutral and Other; in the last case naming their own interpretation. The participation was optional. In total we had 67 participants ( 28 female, 38 male, $1 \mathrm{n} / \mathrm{a})$ ranging in age from 15 to $72(\mathrm{MD}=26.9$, $\mathrm{SD}=12.5)$. We achieved our best results with our expression of sadness (Sadness $1=95.5 \%$, Sadness $2=91.0 \%$ ) and our worst results with joy (Joy $1=73.1 \%$, Joy $2=74.6 \%$ ), see Table I. Overall we had very satisfying recognition ratios.

For Anger wrong labels mostly resulted from our very strict handling of the option "Other". When an expression was labeled with "Other" we rated it as a misclassification, though in most of these cases concerning Anger, the participants just used similar terms. Fear 1 was often interpreted as "shocked" and the expressions of Joy as "cheering". In some cases Joy was labeled as neutral and to our surprise Joy 2 was sometimes labeled as Anger. According to de Meijer the final position of our movement in Joy 2 (also see Figure 1) could be associated with Anger and impact the overall perception of this expression. That Fear 2 was sometimes wrongly labeled as Sadness did not come as a surprise. As mentioned before the underlying posture of Sadness 1 and Fear 2 is quite similar. But only in very few cases Sadness was misclassified as Fear. The few other interpretations of Sadness had nothing in common.
TABLE I

RECOGNITION RATIOS FOR THE EIGHT EXPRESSIONS OF THE PRE-TEST

\begin{tabular}{|c||c|c|c|c|}
\hline & Anger & Fear & Sad & Joy \\
\hline Verison 1 & $82.1 \%$ & $82.1 \%$ & $95.5 \%$ & $73.1 \%$ \\
\hline Version 2 & $94.0 \%$ & $85.1 \%$ & $91.0 \%$ & $74.6 \%$ \\
\hline
\end{tabular}

Some participants indicated as comments that they were able to recognize a particular emotion because of one dominant modality, such as the crying sound. With this in mind we measured the expressivity of the single expressional cues, to see which differences occur in their perception

\section{THE EXPERIMENT}

\section{A. Method}

We separated the expressions into their cues: Body Movements, Sounds and Eye Colors. So we had eight different Body Movements, eight different Sounds and four different Eye Colors, in total 20 different expression cues. In this study the users were not asked to label the cues with a specific emotion, instead they had to assign a specific value within the Pleasure-Arousal-Dominance (PAD) model created by Mehrabian and Russell [11]. We decided to use the PAD model as it is firstly often used to measure the affective value of facial expressions, speech, and even to classify products. Secondly, there is a validated language independent questionnaire available. We used Bradley and Lang's selfassessment manikin (SAM) [4] with nine pictures for each dimension. We asked the participants also to name one or two emotions they associated with a particular expression, after they had assigned the pictures. Finally, the PAD-based questionnaire allows us to investigate the complementary contributions of the single modalities to the dimensions of Pleasure, Arousal and Dominance.

\section{B. Participants}

We had 42 voluntary participants (33 male, 9 female), mostly students in Computer Science, ranging in age from 19 to 29 ( $\mathrm{MD}=24.4, \mathrm{SD}=7.2$ ). They were divided equally in four groups. Each group was only presented with half of the cue set (four Body Movements, four Sounds, two Eye Colors) in two different orders, to avoid learning and ordering effects. Thus each expression cue was at least rated 20 times but not more than 22 times. In most cases two participants at once watched the robot perform and filled in the questionnaire after each action. When playing a sound or 


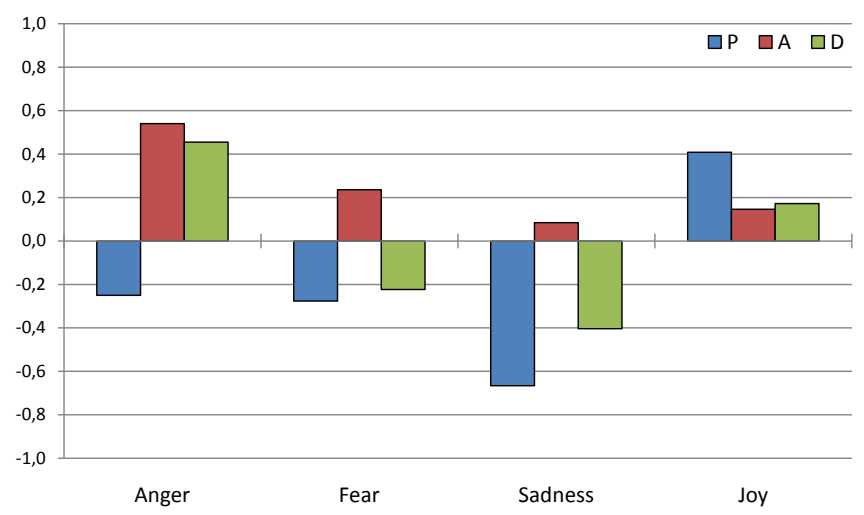

Fig. 2. Results for the PAD measurements for all expression cues grouped by Anger, Fear, Sadness, and Joy.

changing the color of its eyes the robot was just standing up straight. The participants could ask to repeat the last action. A session took about 10 minutes.

\section{Results}

In the following we first analyze how the different expression cues differ within the four groups of emotions (i.e. Anger, Fear, Sadness, and Joy). Thus, we put all PAD measures of the expression cues (i.e. Body Movement, sound, and eye color) of a specific emotion together (see Figure 2) and use the one-way analysis of variance (ANOVA) combined with Bonferroni as post-hoc test for a two-sided pairwise comparison.

The comparison of the four emotions with ANOVA revealed significant differences in the PAD perception among Pleasure (P), Arousal (A), and Dominance (D).

ANOVA revealed for the Pleasure (P) dimension $(F(3,416)=116.2, p<0.001)$ significant differences. The pairwise Bonferroni post-hoc analysis revealed significant differences between all emotions (all $p<0.001$ ) except between Anger and Fear $(p=1.0)$, which was expected as literature states that Anger and Fear cannot be distinguished from each other within the Pleasure dimension.

The ANOVA test for the Arousal (A) dimension revealed significant differences $(F(3,416)=13.9, p<0.001)$. The pairwise Bonferroni post-hoc analysis revealed significant differences for Anger compared with Fear, Sadness, and Joy (all $p<0.001$ ). In contrast to Fear, Sadness and Joy compared with each other which did not reveal significant differences within the Arousal dimension.

And finally, also the ANOVA test for the Dominance (D) dimension revealed significant differences $(F(3,416)=$ $58.3, p<0.001)$. The pairwise Bonferroni post-hoc analysis revealed significant differences between all emotions (all $p<0.001)$ except between Fear and Sadness $(p=0.08)$.

Except Fear and Sadness, which only differs significantly within the Pleasure dimension, all other combinations differ significantly at least within two dimensions.

Our measurements for Anger (-P+A+D, Hostile), Fear ($\mathrm{P}+\mathrm{A}-\mathrm{D}$, Anxious $)$, and Joy $(+\mathrm{P}+\mathrm{A}+\mathrm{D}$, Exuberant $)$ fall into

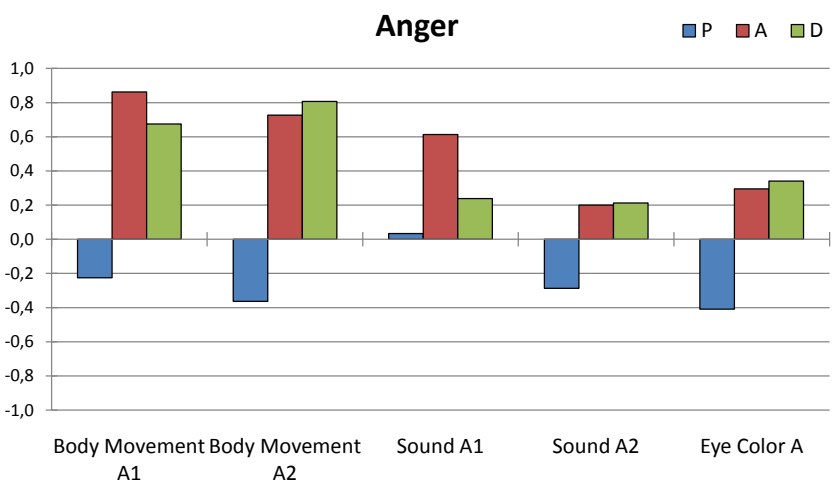

Fig. 3. The PAD measurements for Anger should be in the Hostile ($\mathrm{P}+\mathrm{A}+\mathrm{D}$ ) octant of the temperament space.

the related octants of the temperament space defined by Mehrabian [10]. Sadness so far is an exception. Russell and Mehrabian [15] defined sadness or depression as Bored (P-A-D), but we measured it overall as Anxious (-P+A-D). We will take a closer look at this discrepancy later in this section.

\section{A. Body Movement, Sound and Eye Color}

The previous analysis revealed promising results. Therefore we took a deeper look into the differences of the single emotional expressions in comparison to the three expression cues Body Movement, Sound, and Eye Color.

\section{B. Anger}

The PAD results for the single expression cues for Anger (see Table II and Figure 3) are all within the related Hostile $(-\mathrm{P}+\mathrm{A}+\mathrm{D})$ octant of the temperament space, except Sound A1. Unfortunately, the gibberish was recognized more as Exuberant $(+\mathrm{P}+\mathrm{A}+\mathrm{D})$. This means that the sound was still recognized as aroused, which is related to the Hostile octant, but the Pleasure dimension was slightly rated as positive. When we look at the single ratings, we notice that half of the participants interpreted the sound as angry, rating the Pleasure negative, while the other half interpreted it as excited, accordingly rating it positive, thus resulting in an almost neutral rating for Pleasure. Pittman and Scherer [14] confirm that sound features correlating with Anger also correlate with forms of Joy. Thus it is comprehensible, why this ambiguous perception occurred. Further tests have to clarify if in combination with Body Movement this ambiguity is resolved, and so this sound is still usable for a expression of Anger. Probably we will create a revised version that has a stronger tendency to Anger.

\section{Fear}

Within the expression cues for Fear, Body Movement F1, Body Movement F2 and Sound F2 were recognized as Anxious (-P+A-D) (see Table II and Figure 4). The Eye Color $F$ was recognized as Docile (+P-A-D). This might be due to the lightening conditions of our laboratory, which resulted in a considerable brighter perception of this color. Valdez 
TABLE II

PAD RESUlTS FOR ANGER, FEAR, SADNESS, AND JOY

\begin{tabular}{|r||c|c||c|c||c|c|}
\hline & $\mathrm{P}$ & $S D$ & $\mathrm{~A}$ & $S D$ & $\mathrm{D}$ & $S D$ \\
\hline Anger & & & & & & \\
\hline Body Movement A1 & -0.23 & 0.50 & 0.86 & 0.17 & 0.68 & 0.36 \\
\hline Body Movement A2 & -0.36 & 0.32 & 0.73 & 0.27 & 0.81 & 0.22 \\
\hline Sound A1 & 0.03 & 0.47 & 0.61 & 0.44 & 0.24 & 0.31 \\
\hline Sound A2 & -0.29 & 0.38 & 0.20 & 0.65 & 0.21 & 0.69 \\
\hline Eye Color A & -0.41 & 0.34 & 0.30 & 0.55 & 0.34 & 0.62 \\
\hline Fear & & & & & & \\
\hline Body Movement F1 & -0.33 & 0.32 & 0.68 & 0.25 & -0.01 & 0.70 \\
\hline Body Movement F2 & -0.74 & 0.27 & 0.75 & 0.21 & -0.90 & 0.21 \\
\hline Sound F1 & -0.13 & 0.24 & 0.08 & 0.41 & 0.14 & 0.33 \\
\hline Sound F2 & -0.35 & 0.27 & 0.31 & 0.49 & -0.18 & 0.52 \\
\hline Eye Color F & 0.18 & 0.42 & -0.69 & 0.38 & -0.19 & 0.44 \\
\hline Sadness & & & & & & \\
\hline Body Movement S1 & -0.73 & 0.29 & 0.52 & 0.31 & -0.65 & 0.45 \\
\hline Body Movement S2 & -0.68 & 0.44 & -0.05 & 0.46 & -0.31 & 0.56 \\
\hline Sound S1 & -0.93 & 0.14 & 0.09 & 0.56 & -0.54 & 0.47 \\
\hline Sound S2 & -0.81 & 0.23 & -0.09 & 0.51 & -0.60 & 0.32 \\
\hline Eye Color S & -0.18 & 0.35 & -0.08 & 0.52 & 0.13 & 0.46 \\
\hline Joy & & & & & & \\
\hline Body Movement J1 & 0.48 & 0.36 & 0.50 & 0.37 & 0.44 & 0.33 \\
\hline Body Movement J2 & 0.14 & 0.52 & 0.59 & 0.23 & 0.47 & 0.43 \\
\hline Sound J1 & 0.49 & 0.13 & -0.03 & 0.45 & -0.18 & 0.36 \\
\hline Sound J2 & 0.83 & 0.22 & 0.24 & 0.48 & 0.28 & 0.27 \\
\hline Eye Color J & 0.16 & 0.38 & -0.52 & 0.41 & -0.10 & 0.23 \\
\hline & & & & & & \\
\hline
\end{tabular}

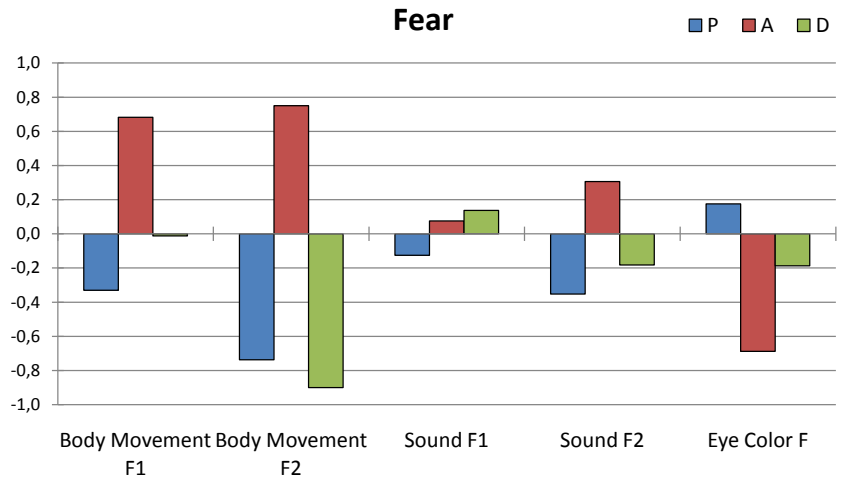

Fig. 4. The PAD measurements for Fear should be in the Anxious (-P+A-D) octant of the temperament space.

[16] mentions that the effect of brightness - in contrast to darkness - is +P-A-D hence reversing our intended effect. This leads to the problem that the perception of color and thus its emotional effect depends a lot from lightening. And second, Sound $F 1$ was recognized as Hostile $(-\mathrm{P}+\mathrm{A}+\mathrm{D})$. This means that the metallic bang sound was perceived more as dominant than as submissive.

\section{Sadness}

The designs of the expression cues for Sadness (which relates to the Bored (-P-A-D) octant in the temperament space) were more challenging (see Table II and Figure 5). Body Movement S2 and Sound S2 were classified as Bored, which is the related octant within the temperament space. But the Eye Color $S$ was classified as Disdainful ($\mathrm{P}-\mathrm{A}+\mathrm{D})$. This means, that the Dominance dimension was

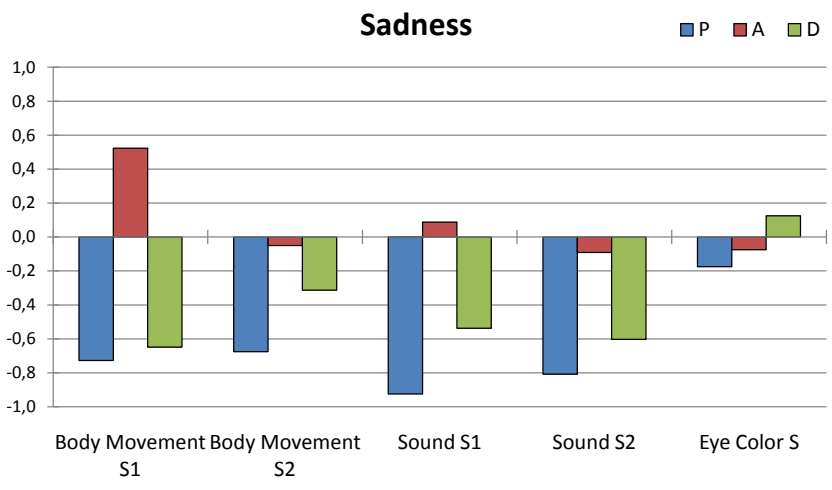

Fig. 5. The PAD measurements for Sadness should be in the Bored (-PA-D) octant of the temperament space.

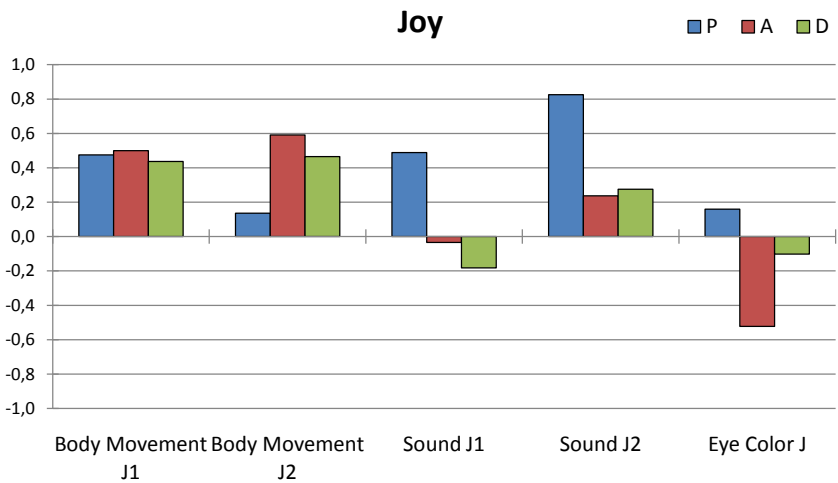

J1

Fig. 6. The PAD measurements for Joy should be in the Exuberant $(+\mathrm{P}+\mathrm{A}+\mathrm{D})$ octant of the temperament space.

wrongly perceived. Body Movement S1 and Sound S1 were both perceived as Anxious (-P+A-P). In contrast to our results of the pre-test, where only few participants labeled Sadness as Fear, this time a lot named the term "fearful" for Body Movement S1. In case of Sound S1 most participants interpreted it as "grief" or "sad". A look at the details shows that participants rated Arousal often very low as well as very high, thus resulting in a rather low mean value. This ambiguous perception of Arousal makes Sound S1 problematic for our further research. Though grief and sadness are related emotions, we need a more definite perception, so we can reliable relate this cue to a definite emotion.

\section{E. Joy}

All expression cues for Joy, except Eye Color J and Sound $J 1$, were perceived as Exuberant $(+\mathrm{P}+\mathrm{A}+\mathrm{D}$ ) (see Table II and Figure 6). The Eye Color $J$ was perceived as Docile (+P-A$\mathrm{D})$, which means first that the arousal level was quite low and second that it was perceived slightly as submissive. And finally Sound JI was also perceived as Docile. Sound JI was using the built-in text-to-speech (TTS) module of the robot to express joy. The voice sounded quite evenly. Thus, it is not surprising that Sound $\mathrm{Jl}$ was perceived as neither aroused nor dominant.

To summarize, in most cases the Eye Color does not 
match the expressivity of the related movements and sounds, except for Anger, where the color red, seems to support the emotional expression quite accurately.

Also when it comes to Sound we have to admit that half of our cues does not match the intended octant. Though all these sounds - except Sound J1 - only differ in one dimension, this considerably changes the perception. For Sound J1 we note that the text-to-speech did not convince the participants.

We are quite content with our Body Movements. Only Body Movement S1 does not fit the target emotion.

\section{Conclusions}

Using psychological findings on the expression and perception of emotions as basis for our expressional design, we were able to achieve in our pre-test similar results to Zecca and colleagues and Beck and colleagues, if not better.

But especially the often confusion of Joy 2 with Anger and the comments of the participants indicating dominant cues, asked for a deeper look. Using the Pleasure-ArousalDominance model and the corresponding SAM test, we were able to measure the affective perception of each single cue. In this way we were able to evaluate the suitability of the different modalities and identify flaws in our design.

First of all, according to our results Eye Colors are not a reliable component for emotional expression. Alone the fact that different lightening conditions might significantly change the perception of the color, makes this modality hard to handle. Only the color red for Anger was able to support its target emotion. For us this means that we will focus our future work on Body Movement and Sound, as the combination of these two modalities into a proper expression is challenge enough.

The fact that our Body Movements achieved the best results in our experiment, suggests that the research so far done for human emotional expression with body movement is applicable for expressions with humanoid robots. According to the PAD results and the comments Body Movement S1 should be reassigned as an expression of Fear rather than an expression of Sadness. Also Body Movement F1 was probably to fast. Strong and fast backward movements are in fact related to Fear (see [12]), but in this case some subjects were startled while watching the expression. It was also labeled more as "shocked" than "afraid", so we will work on a revision that is less extreme.

Half of our Sounds were appropriate for their target emotion. So the approach to use modified human or animal noises, as well as music, seems practical. On the other hand half of our Sounds did not match the expected octant. We need to get more seriousness in the gibberish voice of Sound $A 1$, to direct the perception more to "angry" than "excited". The crying sound of Sound S1 was too extreme. The participants were torn between "grief" and "sadness" and no common perception of Arousal could be achieved. Sound S2 was similar but less extreme and hence matched its target emotion. The metallic bang of Sound Fl seemed fitting in combination with the high speed of Body Movement F1, but the experiment revealed that as a single cue it has not much expressivity especially none for Fear. Considering the fact, that we want to revise the corresponding Body Movement, a replacement is the most reasonable solution. As mentioned before in section IV the attempt to use the integrated textto-speech module of Nao was not successful. The voice was to monotonous and not able to properly stimulate the participants. Most likely we will replace this cue with a recorded and modified human sound or a appropriate piece of music.

Considering these findings we will revision our Body Movements and Sounds so they better match their target emotion. Our library consisting of the revisioned and future expressions will be available at http://hcm-lab.de/projects/shr.

\section{ACKNOWLEDGMENTS}

We would like to thank our former students Ferenc Bujtor and Jürgen Weichenmeier for their work on the emotional expressions.

\section{REFERENCES}

[1] J. A. Bachorowski and M. J. Owren. Sounds of emotion: production and perception of affect-related vocal acoustics. Ann N Y Acad Sci, 1000:244-265, December 2003.

[2] A. Beck, L. Cañamero, and K.A. Bard. Towards an affect space for robots to display emotional body language. In IEEE RO-MAN, 2010.

[3] R. T. Boone and J. G. Cunningham. Children's expression of emotional meaning in music through expressive body movement. Journal of Nonverbal Behavior, 25:21-41, 2001.

[4] M. M. Bradley and P. J. Lang. Measuring emotion: The selfassessment manikin and the semantic differential. Journal of Behavior Therapy and Experimental Psychiatry, 25(1):49 - 59, 1994.

[5] C. L. Breazeal. Designing Social Robots. The MIT Press, 2002.

[6] M. Coulson. Attributing emotion to static body postures: Recognition accuracy, confusions, and viewpoint dependence. Journal of Nonverbal Behavior, 28:117-139, 2004.

[7] B. de Gelder. Why bodies? Twelve reasons for including bodily expressions in affective neuroscience. Philosophical Transactions of the Royal Society B: Biological Sciences, 364(1535):3475-3484, December 2009.

[8] K. Grammer and E. Oberzaucher. The reconstruction of facial expressions in embodied systems: New approaches to an old problem. In ZiF Mitteilungen, volume 2, pages 14-31, 2006

[9] K. Kühnlenz, S. Sosnowski, and M. Buss. The impact of animallike features on emotion expression of robot head eddie. Advanced Robotics, 24(8-9), 2010.

[10] A. Mehrabian. Pleasure-arousal-dominance: A general framework for describing and measuring individual differences in temperament. Current Psychology, 14:261-292, 1996.

[11] A. Mehrabian and J. A. Russell. An Approach to Environmental Psychology. The MIT Press, April 1974.

[12] M. Meijer. The contribution of general features of body movement to the attribution of emotions. Journal of Nonverbal Behavior, 13:247268, 1989.

[13] J. Monceaux, J. Becker, C. Boudier, and Alexandre Mazel. Demonstration: first steps in emotional expression of the humanoid robot nao. In Proceedings of the International Conference on Multimodal interfaces, ICMI-MLMI, 2009.

[14] J. Pittman and K. R. Scherer. Vocal expression and communication of emotions. In Handbook of Emotions. The Guildford Press, 1993.

[15] J. A. Russell and A. Mehrabian. Evidence for a three-factor theory of emotions. Journal of Research in Personality, 11(3):273-294, September 1977.

[16] P. Valdez and A. Mehrabian. Effects of color on emotions. J Exp Psychol Gen, 123(4):394-409, December 1994.

[17] M. Zecca, Yu. Mizoguchi, K. Endo, F. Iida, Y. Kawabata, N. Endo, K. Itoh, and A. Takanishi. Whole body emotion expressions for kobian humanoid robot - preliminary experiments with different emotional patterns. In IEEE RO-MAN, 2009. 